\title{
Mecanismos de evaluación y seguimiento en planes de ordenación del territorio de Andalucía, España
}

\author{
Monitoring and Evaluation Mechanisms in Spatial Planning in \\ Andalusia, Spain

\section{Sergio Segura Calero ${ }^{1}$} \\ Belén Pedregal Mateos ${ }^{2}$
}

Para citar este artículo, utilice el nombre completo así:

Segura Calero, S \& Pedregal Mateos, B. (2014) Mecanismos de evaluación y seguimiento en planes de ordenación del territorio de Andalucía, España. Perspectiva Geográfica, 19(2), 357-378

\section{Resumen}

Los mecanismos de evaluación y seguimiento de los planes de ordenación del territorio comprenden todos los procesos previstos y regulados en estos planes con la función de favorecer su evaluación, seguimiento y retroalimentación. En este trabajo se analizan los mecanismos que forman el sistema de seguimiento y evaluación de los planes de ordenación del territorio andaluces, como parte del sistema de gobernanza territorial de esta región meridional española, a partir del examen de los propios planes, la normativa vigente y aplicación de la misma por parte de los órganos competentes. Hasta qué punto su implementación hasta la fecha ha resultado exitosa en el caso de Andalucía también será objeto

1 Licenciado en Geografía, Universidad de Sevilla. Personal investigador en formación, becario FPU del Ministerio de Educación, Departamento de Geografía Humana, Universidad de Sevilla. Observatorio de la Diversidad, la Cohesión y el Desarrollo Territorial de Andalucía (ODTA). Grupo de Investigación Estructuras y Sistemas Territoriales (GIEST). ssegura@us.es

2 Doctora en Geografía, Universidad de Sevilla. Profesora titular, Departamento de Geografía Humana, Universidad de Sevilla. Observatorio de la Diversidad, la Cohesión y el Desarrollo Territorial de Andalucía (ODTA). Grupo de Investigación Estructuras y Sistemas Territoriales (GIEST). bpedregal@us.es 
de análisis crítico, puesto que el desarrollo establecido, tanto en la normativa como en la práctica en esta materia, presenta algunas carencias y dificultades que se agravan por la limitación de recursos en el contexto de crisis económica actual.

Palabras clave: Andalucía, gobernanza territorial, mecanismos de seguimiento, ordenación del territorio, sistemas de evaluación y seguimiento.

\section{Abstract}

Monitoring and evaluation mechanisms of spatial plans are regulated processes in these plans to promote their evaluation, monitoring and feedback. In this paper we analyze the mechanisms for Andalusia spatial planning, as part of the territorial governance system of this Spanish southern region, through an examination of the plans \& regulations and their implementations by the competent bodies and agencies.

Keywords: Andalusia, territorial governance, monitoring mechanisms, spatial planning, monitoring and evaluation systems. 


\section{Introducción}

\section{Contextualización de Andalucía y} de la ordenación del territorio en España

En el contexto actual, donde el uso racional de los recursos y el paradigma del desarrollo territorial sostenible resultan cada vez más evidentes y necesarios, la ordenación del territorio, entendida como política integral de planificación física a escala regional y subregional, cobra un papel fundamental. Los problemas detectados en esta materia son relevantes en muchos países $\mathrm{y}$, por tanto, los mecanismos de evaluación y la capacidad de adaptación de los planes o instrumentos de ordenación del territorio son una parte imprescindible para valorar la eficacia y el alcance real de los objetivos propuestos por estos mismos. En este artículo abordaremos el caso de la comunidad autónoma de Andalucía en España. Por tanto, resulta necesaria una breve introducción de Andalucía, del significado y origen de la ordenación del territorio vigente en España y en la región andaluza, así como la explicación de conceptos y fundamentos teóricos que enmarcan este tema.



Figura 1. Mapa de localización y distribución de la población en Andalucía

Fuente: elaboración propia a partir de Datos Espaciales de Referencia de Andalucía (DERA, 2014) y Padrón de habitantes 2013. Instituto de Estadística y Cartografía de Andalucía.

Andalucía (Figura 1) es la región más meridional de la península ibéri- ca y presenta un peso relevante en el conjunto de España por ser la comu- 
nidad autónoma más poblada, con casi ocho millones y medio de habitantes, según el Censo de población de 2011, y la segunda en extensión con $87.597 \mathrm{~km} 2$. Se trata de una de las regiones más grandes de Europa, superando en tamaño y población a muchos de los Estados que componen la Unión Europea. Históricamente suele tildarse como una región de encrucijada entre continentes y mares, un territorio propicio para el intercambio de bienes y culturas con largo recorrido, incluyendo sus lazos con Latinoamérica. Este estatus se consolida todavía más por la confluencia en Andalucía de tres grandes ejes asociados a grandes estrategias regionales económicas de la Unión Europea: arco mediterráneo, arco atlántico y diagonal continental de Europa occidental. Por tanto en Andalucía es notoria la relación histórica e intensa de su población con el territorio y su gran diversidad geográfica. Sin embargo, se caracteriza por tratarse de una región desfavorecida económicamente dentro del conjunto de España y de Europa, con unos ingresos per cápita inferiores a la media de la Unión (Comisión Europea-CE-, 2011). Esto supuso la llegada de múltiples mecanismos y ayudas como los Fondos Europeos de Desarrollo Regional. En la actualidad Andalucía ha superado la situación de objetivo prioritario de la política regional europea pero, a su vez, ha sido duramente golpeada por la crisis económica. Un reflejo claro de la diversidad mencionada anteriormente es el interesante sistema de ciudades que existe en Andalucía, sustentado por una densa red de históricas agrociudades, actuales ciudades medias del sistema urbano, que presenta un gran potencial para favorecer el policentrismo establecido como objetivo en la Estrategia Territorial Europea, así como la cohesión y el equilibrio territorial.

La ordenación del territorio en España como cualquier otra política pública se ve afectada por las regulaciones y directivas europeas. Sin embargo, no existe normativa europea específica en esta práctica ya que las competencias en esta materia pertenecen a los propios estados miembros. En España son las regiones, las denominadas comunidades autónomas, las que tienen estas competencias. Si bien es cierto que existen directivas europeas y planificaciones de competencia estatal sectoriales o transversales que inciden de forma directa en la ordenación del territorio; algunas con gran calado como por ejemplo la planificación de grandes infraestructuras, la ambiental o la hidrológica. Las grandes orientaciones para las políticas sectoriales con impacto territorial en Europa y el fomento de la colaboración e integración de actuaciones se encuentran en la Estrategia Territorial Europea (Comisión Europea -CE-, 1999), 
como documento de referencia para la ordenación del territorio y el desarrollo regional equilibrado y sostenible en la Unión Europea desde 1999.

La ordenación del territorio aparece como competencia exclusiva de las comunidades autónomas en el artículo 148.1 de la Constitución Española de 1978, junto con las competencias de urbanismo y de vivienda con las que está estrechamente vinculada. Es así como esta política comienza sus pasos en España desde la década de los 80 , lo que ha supuesto un largo proceso de casi 20 años para la consolidación normativa de esta política desde la primera ley de 1983 en Cataluña hasta la de 2001 en Extremadura. Por tanto, se deduce que ha sido difícil su implementación, pero hoy día ya puede afirmarse que es una experiencia bastante establecida, puesto que casi todas las comunidades autónomas de España, aunque con diferencias, han aprobado o se encuentran tramitando planes formales de ordenación del territorio que son los instrumentos que materializan esta política (Benabent, 2009, 2012).

Sin embargo, la ordenación del territorio ha sido considerada menos relevante que el urbanismo en el contexto español y andaluz. Los aspectos inmediatos económicos y locales de la planificación urbanística y sus problemas asociados siempre han tenido un papel protagonista.
Este distanciamiento entre la ordenación del territorio y urbanismo y la no exclusividad autonómica de competencias en materia económica han propiciado que la Ley de Ordenación del Territorio de Andalucía y en general las leyes de ordenación del territorio en España, presenten una orientación de planificación física del territorio, aunque lógicamente también relacionada con objetivos económicos, como ocurre en muchos de los Estados federales europeos (Alemania, Austria o Suiza) y no de corte fuertemente económico como Francia y la tradición planificadora económica española. Aunque es importante mencionar que la Ley de Ordenación del Territorio de Andalucía está en origen estrechamente vinculada al planeamiento urbanístico y poco a poco los planes de ordenación territoriales han asumido los conceptos de estructura general y orgánica del territorio (Zoido, 2010). En España los planes muestran una doble naturaleza, por un lado como impulsores de transformaciones territoriales y elementos dinamizadores, a la vez que por otro regulan y restringen usos y actuaciones sobre el territorio (Benabent, 2009). Además presentan dos escalas fundamentales bien diferenciadas, que se corresponden con sendos instrumentos de ordenación previstos por las legislaciones autonómicas españolas (Feria, Rubio \& Santiago, 2005; Hildenbrand, 2006): 
a) Instrumentos de ordenación del territorio de ámbito regional. En algunas comunidades autónomas reciben el nombre de planes, aunque generalmente en las leyes autonómicas se denominan directrices puesto que es su cometido básico. Presentan por lo general un carácter más estratégico. También estos planes pueden ser reconocidos como estrategias de ordenación territorial. En el caso andaluz se denomina Plan de Ordenación del Territorio de Andalucía (POTA), vigente desde 2006.

b) Instrumentos de ordenación del territorio de ámbito subregional. Estos instrumentos más concretos están vinculados a territorios de escalas intermedias entre la planificación regional y la local (provincial, comarcal o supramunicipal) y no son contemplados en todas las leyes autonómicas, puesto que algunas comunidades autónomas no han considerado necesaria la implementación de esta figura por contemplar una única provincia en su región. Normalmente el nombre que reciben estos instrumentos es el de plan territorial subregional o parcial, aunque también aparecen como directrices en casos que coinciden con instrumentos de mayor contenido normativo. En Andalucía estos reciben el nombre de plan de ordenación del territorio subregional y actualmente se cuenta con 22 planes entre los que se encuentran aprobados (17), en trámite (2) y en redacción (3).

Recientemente, a estos planes debe añadirse una nueva figura de planeamiento incorporada en la Ley de Ordenación del Territorio de Andalucía a través del Decreto-ley 5/2012, de 27 de noviembre, de medidas urgentes en materia urbanística y para la protección del litoral de Andalucía. Esta modificación incorpora a las dos figuras de ordenación territorial anteriores el Plan de Protección del Corredor Litoral de Andalucía, cuyo documento inicial ya se ha publicado con el objetivo de poder intervenir en la protección del ámbito litoral e incentivar el cumplimiento de las determinaciones del POTA. Su ámbito afecta al menos a los primeros $500 \mathrm{~m}$ de la zona de influencia del litoral y aquellas otras zonas necesarias para cumplir los objetivos originales del plan. Por tanto, este plan queda establecido por la Ley de Ordenación del Territorio de Andalucía en el marco del POTA y presenta un carácter vinculante hacia los planes de ordenación del territorio subregionales que afecta.

\section{Mecanismos de evaluación y segui- miento en planes de ordenación territorial: definición y marco teórico}

Una vez contextualizada la situación de la ordenación del territorio en Andalucía y de sus planes, parece apro- 
piado comenzar con la definición del concepto esencial tratado en este trabajo. Primordialmente ent-endemos como mecanismos de evaluación y seguimiento de los planes de ordenación del territorio todos los procesos previstos en estos planes que tienen que ver con la retroalimentación del mismo y, por tanto, con la finalidad de recabar información continua, disponible a corto plazo o en tiempo real, para el control y la toma de decisiones sobre los efectos de dichos instrumentos de ordenación del territorio, así como para tener en consideración los cambios que se hayan producido en este último. En consecuencia, asumimos la más que consolidada crítica posmodernista a la ordenación del territorio del modelo clásico de planificación basado en un proyecto y no en un proceso continuo, como ciclo en el tiempo (Juaristi, 2009). Estos mecanismos, si se encuentran institucionalizados con fundamentos legales, pueden llegar a formar un sistema de seguimiento $y$ evaluación que hace referencia al conjunto organizado de leyes, normativas, instituciones, organismos, metodologías y procesos que hacen posible la supervisión y la adaptación de los planes de ordenación territorial a los cambios en el espacio y en el tiempo. Por tanto, este sistema puede facilitar el ajuste de los planes de ordenación del territorio a sus objetivos y además forma parte fundamental de lo que podríamos reco- nocer como sistema de gobernanza territorial, y que definiremos a continuación, tomando como referencia, entre otras, la experiencia pionera en Europa de la Estrategia Territorial de Navarra (Collado, 2004; Munarriz, 2011; Zarraluqui, 2003).

La definición e implementación de un sistema de evaluación y seguimiento se sitúa en el marco teórico de la gobernanza territorial. Farinós y Romero (2008) ya destacaron que el concepto específico de gobernanza territorial, y sus múltiples dimensiones (ver también ESPON Project 2.3.2, 2007), es todavía más complejo que el de simple gobernanza. Estos autores entienden el concepto de gobernanza para los territorios como un nuevo buen gobierno donde el Estado tiene un papel fundamental, la democracia es más sofisticada y la sociedad civil forma parte del proceso político como protagonista $\mathrm{y}$, por tanto, la participación pública es un elemento clave. En consecuencia, son necesarios, entre otras cosas, nuevos procedimientos para llevar a cabo las políticas, y también facilitar mecanismos de control que permitan el monitoreo y evaluación de las mismas. Sin embargo, se advierte que la sociedad no está igualmente implicada en los asuntos públicos en distintos territorios y puede dar lugar a que la nueva gobernanza participada responda únicamente a los grupos con poder. A pesar de es- 
tas críticas y dificultades detectadas en su implementación real, algunos autores han reconocido el spatial planning o la nueva ordenación del territorio o nueva planificación territorial estratégica como política predilecta para desarrollar la cooperación interadministrativa (Massiris, 2002; Feria et al. 2005; Romero \& Farinós, 2011).

Además del seguimiento propiciado por los mecanismos de participación pública, los sistemas de indicadores sobre los objetivos políticos y territoriales contemplados en los planes constituyen otro instrumento importante de evaluación. Estos instrumentos tienen un notable recorrido en el caso de las políticas ambientales $\mathrm{y}$, en los últimos años, un gran impulso propiciado por los objetivos de cohesión social y territorial, así como por el objetivo más general de desarrollo sostenible.

Con el principio de cohesión territorial, la Unión Europea busca la articulación física de las regiones, la equidad territorial vinculada a la accesibilidad, al desarrollo personal y también asentar la identidad territorial de las personas con un proyecto de vida común. En ese sentido, los sistemas de indicadores de cohesión territorial, de carácter tanto cuantitativo como cualitativo, resultan un instrumento adecuado para orientar y evaluar los progresos de las políticas relativas a este concepto y para facilitar el análisis prospectivo y de escenarios (FernándezTabales, Pedregal, Rodríguez, Pita \& Zoido, 2009). Las experiencias de la Agencia Europea de Medio Ambiente y otros proyectos como los impulsados por el observatorio territorial europeo ESPON $\backslash$ ORATE o el Urban Audit son también referentes importantes en el mismo sentido. Por tanto, dentro de los mecanismos regulados de evaluación y seguimiento de planes, políticas y programas, destaca el uso de estos sistemas de indicadores.

En el caso de los indicadores de desarrollo sostenible, en los últimos años estos están siendo concebidos como el resultado del proceso de interacción sociopolítica, entre instituciones y sociedad, donde las oficinas nacionales de estadística tienen un papel destacado. Garnåsjordet, Aslaksen, Giampietro, Funtowicz, y Ericson (2012) expresan esta nueva concepción a través de las narrativas que subyacen tras los indicadores. No se trata de una mera selección técnica estadística sino que los baremos elegidos responden a intereses sociales, y esto es crucial a la hora de establecer los valores normativos del proceso de generación de estos datos. La elección participada de indicadores de evaluación y seguimiento resulta, pues, una propuesta necesaria en este nuevo marco teórico. 
En los próximos epígrafes se analizarán los mecanismos de evaluación y seguimiento en los nuevos planes de ordenación del territorio en Andalucía a partir del examen de los propios planes, la normativa vigente y aplicación de la misma por parte de los órganos competentes. Además se revisará el papel que cumplen las distintas instituciones y organismos encargados de supervisar y realizar las evaluaciones y el seguimiento de los planes de ordenación del territorio en Andalucía. Estos organismos son, en ocasiones, determinaciones de los propios instrumentos de planificación, como se muestra en los siguientes apartados.

\section{Mecanismos de evaluación y se- guimiento en planes de ordenación territorial andaluces}

\subsection{El sistema de gobernanza terri- torial andaluz}

Como ya se ha comentado, la Ley de Ordenación del Territorio de Andalucía de 1994, recientemente modificada por la adopción de medidas cautelares urgentes en el ámbito del litoral en noviembre de 2012, es la normativa de referencia en ordenación del territorio para la comunidad autónoma. Por tanto, merece la pena empezar por su análisis. En ella, en su artículo 11, se recogen los contenidos de los planes de ordenación del territorio de Andalucía: el Plan Regional (POTA), el Plan de Protección del Corredor Litoral de Andalucía y los planes de ordenación del territorio subregionales. Por tanto, podemos empezar aquí con el origen de los mecanismos de seguimiento y evaluación previstos para la planificación territorial por la Ley. En todos aquellos planes se exige como contenido las previsiones para su desarrollo, seguimiento y ejecución. Además, en los planes deben ser explicitados los criterios de periodicidad y contenidos necesarios para la elaboración de memorias de gestión del plan. Como otro de los grandes mecanismos de control de la planificación, la Ley menciona comisiones de redacción de los planes necesarias para que faciliten la cooperación y coordinación en materia de competencias del plan. Asimismo, la Comisión de Ordenación del Territorio y Urbanismo de Andalucía y sus comisiones provinciales deben informar, de acuerdo con la Ley, sobre los planes de ordenación del territorio con carácter previo a su aprobación y también para el proceso de revisión y para el de modificación de los mismos. Por último, el desarrollo del Sistema de Información Territorial de Andalucía también es recogido en esta Ley como elemento básico para la evaluación territorial (Tabla 1). 
Tabla 1. Mecanismos de evaluación y seguimiento para incorporar en la normativa de planes de ordenación del territorio en Andalucía según la Ley de Ordenación del Territorio de Andalucía

\begin{tabular}{ll|}
\hline CONTENIDOS: \\
$\checkmark \quad$ Previsiones para su desarrollo, seguimiento y ejecución (Artículos. 7. $k$,11.f y 43.g, LOTA) \\
$\checkmark \quad$ Criterios de periodicidad y contenido de memorias de gestión (Artículos. 7. l, LOTA) \\
\hline ÓRGANOS: \\
$\checkmark \quad$ Comisiones de redacción (coordinación y cooperación interadministrativas) (Art. 8 y 13, LOTA) \\
$\checkmark \quad$ Comisión de Ordenación del Territorio y Urbanismo de Andalucía y comisiones provinciales con \\
$\quad$ el deber de informar previamene la aprobación, revisión o modificación de los planes de \\
$\quad$ ordenación del territorio. (Art. 34 y 35, LOTA) \\
\hline INSTRUMENTOS: \\
$\checkmark \quad$ Sistema de Información Territorial de Andalucía (Art. 33, LOTA)
\end{tabular}

Fuente: elaboración propia a partir de la Ley de Ordenación del Territorio de Andalucía de 1994.

Como veremos más adelante, el alcance de todos estos mecanismos ha tenido, hasta el momento, poco calado. Además, a estos mecanismos debemos añadir la creación, comúnmente mediante el mismo decreto de aprobación definitiva del plan, de una comisión de seguimiento formada por representantes de todos los organismos, instituciones y agentes sociales interesados. Entre las funciones de esta comisión se encuentra el deber de informar las actualizaciones y los informes de seguimiento del plan previstos en la normativa de los planes.

Como ya se ha mencionado anteriormente, las competencias en ordenación del territorio en España son exclusivas para las comunidades autónomas. Pero la Estrategia Territorial Europea ya recomienda la ob- servación y evaluación del desarrollo territorial y sus consecuencias, así como la necesidad de producir información a escala comunitaria con acuerdos sobre criterios e indicadores para poder crear escenarios a largo plazo, sin mermar las previsiones a corto y medio plazo. Sin embargo, existen directivas europeas y planificaciones de competencia estatal sectoriales o transversales que inciden de forma directa en la ordenación del territorio. Concretamente es desde la planificación ambiental donde se han hecho mayores esfuerzos en la Unión Europea con la intención de incorporar consideraciones ambientales y mecanismos de evaluación y seguimiento en políticas, planes y programas. Esto se ha llevado a cabo a través de la evaluación ambiental estratégica (directamente conectada a la evaluación de 
impacto ambiental estratégica). Con esta intención se elaboró la Directiva Europea 2001/42/CE del Parlamento y del Consejo, de 27 de junio de 2001, relativa a la evaluación de los efectos de determinados planes y programas en el medio ambiente, $\mathrm{y}$ también conocida como Directiva de Evaluación Ambiental Estratégica. En esta Directiva encontramos algunos de los principios fundamentales para la incorporación de las medidas de seguimiento y control en los planes que inciden directamente sobre el medioambiente, como los aquí tratados. Además, entre sus consideraciones, la Directiva advierte de la necesidad de unos requisitos de procedimiento comunes en la Unión Europea a la hora de elaborar sistemas de evaluación medioambiental y destaca la importancia de dedicar tiempo para la participación y la expresión de opiniones al respecto.

La citada Directiva fue traspuesta en España mediante la Ley 9/2006, de 28 de abril, sobre evaluación de los efectos de determinados planes y programas en el medio ambiente; y se incorpora en la legislación andaluza a través de la Ley 7/2007 de 9 de julio, de Gestión Integrada de la Calidad Ambiental (GICA). Todas estas normativas de trasposición mantienen la esencia y contenidos de la Directiva en relación con el tema que aquí tratamos. La cuestión de la evaluación y el seguimiento se esta- blece para los planes de incidencia en el medioambiente, como los planes de ordenación del territorio, a través de la incorporación de un Informe de Sostenibilidad Ambiental en la memoria ambiental del plan. Este Informe debe ser elaborado por el órgano promotor y contener una descripción de las medidas previstas para el seguimiento y control de los efectos significativos de la aplicación de los planes y programas. Está previsto, y así se viene realizando hasta la fecha, que la Administración ambiental elabore y tramite un documento de referencia que establezca la amplitud y nivel de detalle del Informe de Sostenibilidad Ambiental que incluya además los criterios ambientales estratégicos e indicadores de los objetivos ambientales y principios de sostenibilidad aplicables en cada caso (Segura \& Pedregal, 2011).

Una vez visto este importante mecanismo de evaluación y seguimiento a través de la legislación ambiental, corresponde ahora trazar el esquema del sistema de gobernanza territorial para la ordenación del territorio en Andalucía, recogiendo de manera comprensiva el conjunto de agentes, normas e instituciones y que podría expresarse, siguiendo el ejemplo de la Estrategia Territorial de Navarra (Munárriz, 2011) a partir de la sistematización propuesta en la Figura 2. 


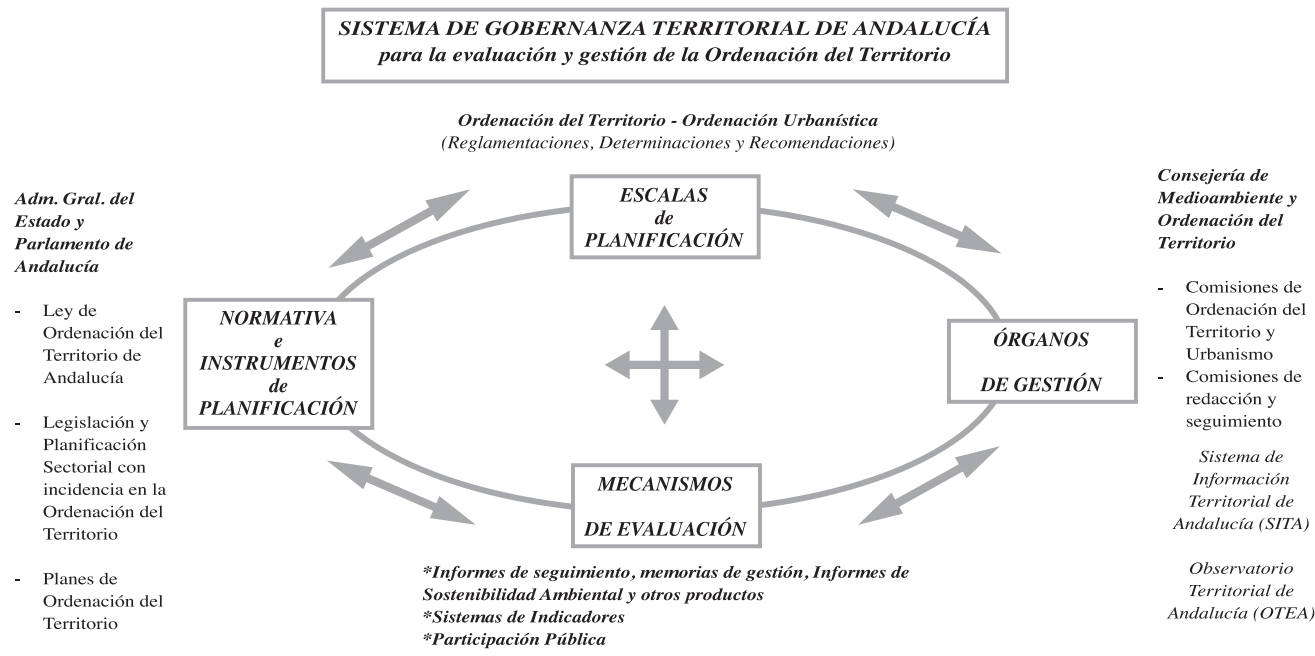

Figura 2. Sistema de Gobernanza Territorial en Andalucía para la ordenación del territorio Fuente: elaboración propia a partir de la consultas a la legislación vigente y Munárriz (2011).

Al sistema todavía habría que agregar los organismos creados con carácter consultivo que están aún desarrollándose como la Comisión Interdepartamental de Valoración Territorial y Urbanística (2008) y la Dirección General de Disciplina Urbanística y el cuerpo de inspectores de ordenación del territorio, urbanismo y vivienda (2005).

Una vez identificados todos estos componentes de forma esquemática, resulta interesante aportar unas valoraciones sobre algunos de estos y otros de los elementos que conforman el sistema. Lo primero que podemos destacar es que el sistema de evaluación y seguimiento, que a su vez forma parte del sistema de gobernanza territorial de Andalucía, quedaría complementado con la aportación de otros entes institucionales, profesionales y sociales pero, salvando los grupos ecologistas y recientemente las organizaciones de defensa de consumidores, no se aprecia casi implicación de la sociedad civil. Desarrollaremos más adelante el papel de la participación pública en nuestro tema. Por otro lado, el Informe del Defensor del Pueblo Andaluz de 2010, constituye una interesante aportación que incluso critica los largos plazos requeridos en los procedimientos de elaboración, tramitación y aprobación de los planes de ordenación del territorio. Sin embargo, en Andalucía $\mathrm{y}$, de acuerdo con las reflexiones de Zoido (2010), aunque ha habido notables mejoras, las dudas y dificultades de la práctica real de esta política son considerables. La continua reformulación de órganos de urbanismo y ordenación del territorio, hasta en cuatro 
ocasiones refundidos, y la fugacidad de cargos en los distintos organismos e instituciones, son un indicador claro de esta afirmación. Además, según el mismo autor, otros actores no parecen demasiado implicados. Esto ocurre, por ejemplo, con la oposición política que solo cuestiona actuaciones, y generalmente cuando algunos conflictos se hacen públicos. Aunque existen, tampoco son muy relevantes las aportaciones particulares de intelectuales, académicos o profesionales. Además, incluso la Federación Andaluza de Municipios y Provincias, que lógicamente debería estar implicada, tampoco se muestra interesada en esta materia. Lo mismo ocurre con los grupos sindicales y con la Confederación de Empresarios de Andalucía que, por su parte, no apoya la regularización restrictiva del suelo.

Por otra parte, es relevante destacar el papel actual de la participación pública en esta materia. Aunque la Ley de Ordenación del Territorio de Andalucía recoge en su exposición de motivos la conveniencia de incorporar la participación pública, y también recoge en su artículo tercero como fundamentos esenciales de la Ley los principios de participación, cooperación y coordinación, entre otros, y por supuesto sin que existan perjuicios competenciales entre administraciones, la participación pública no se establece como un mecanismo de seguimiento o evaluación del plan. Lo cierto es que algunos auto- res han destacado que la participación es entendida como un procedimiento pasivo y de información pública que apenas ha cambiado desde la primera legislación urbanística con la Ley del Suelo de 1956 (Benabent, 2009 y 2012). Esto es así puesto que según la Ley de Ordenación del Territorio de Andalucía solamente existe periodo de participación, de al menos dos meses, una vez formulado el plan, en la fase de avance, y otro periodo igual una vez que ya el plan ha sido redactado, antes de su aprobación definitiva. El ejemplo de buenas prácticas en materia de participación de la Estrategia Territorial de Navarra y sus planes subregionales es siempre citado, aunque se trata de un caso aislado (Collado, 2004; Munárriz, 2011; Zarraluqui, 2003). Como bien define Zarraluqui (2003), se diferencia por su énfasis en el benchmarking (uso de comparadores), no encerrarse en las fronteras regionales, el uso de objetivos y principios como espina dorsal del trabajo, el uso de indicadores territoriales y la participación pública, incluso durante el proceso de formulación del plan.

\subsection{Mecanismos de evaluación y se- guimiento contemplados en los con- tenidos de los planes andaluces}

Después de haber hecho este reconocimiento de normativas, agentes, instituciones y organismos del sistema de gobernanza territorial en An- 
dalucía, que intervienen también en la evaluación y seguimiento de los planes de ordenación del territorio, se presenta ahora la revisión de los contenidos propios de los planes en materia de evaluación y seguimiento. Antes es fundamental recordar que el sistema de planeamiento territorial andaluz integra y relaciona todas las políticas de planificación con incidencia territorial de forma bastante coherente, teniendo en cuenta la complejidad y las múltiples escalas de planificación, y trata de evitar el abuso de poder sobre el territorio de la planificación urbanística (Hildenbrand, 2006, 2009).

El POTA, como plan de ordenación para el conjunto regional andaluz, tomando como referencia las distintas leyes ya mencionadas, expresa una serie de recomendaciones sobre la evaluación estratégica de planes y programas. Por tanto, el POTA tiene la Directiva de evaluación ambiental mencionada como una de sus referencias en sus Estrategias de Desarrollo Territorial y en determinaciones, normativas y recomendaciones relativas a esta materia, así como criterios generales sobre seguimiento y evaluación de este plan regional y de los planes de ordenación territorial subregionales para los que se exige la identificación de medidas e indicadores de seguimiento y evaluación. El desarrollo de estos contenidos y medidas es derivado, para el caso del
POTA, hacia otros organismos que el propio instrumento genera. Estos instrumentos son el Sistema de Información Territorial de Andalucía, ya expresado en la misma Ley, y el Observatorio Territorial de Andalucía. Además el POTA menciona la necesidad de sistemas de información territorial municipales, a ser posible coordinados con otras iniciativas como las Agendas 21 locales. Por otra parte, se incluyen dentro del POTA consideraciones concisas sobre el sistema de indicadores a emplear (POTA, 2007, pp. 163-164).

De forma resumida, los indicadores deben poseer validez científica, su ámbito de cálculo debe ser regional, sus tendencias deben servir para obtener conclusiones y deben ser también evaluados y actualizados anualmente, entre otras consideraciones. Además se diferencian entre dos grandes bloques básicos de indicadores, unos sobre estructura territorial y otros sobre sostenibilidad. El primero se relaciona con indicadores sobre objetivos sociales y económicos y los destinados a las medidas de consecución del Plan. El segundo reúne variables relacionadas con el análisis de la sostenibilidad física del modelo territorial y, por tanto, aglutina indicadores de sostenibilidad y también de gestión del plan. La normativa del POTA también recoge la necesidad de la memoria de gestión del plan periódica relativa al desarrollo y aplica- 
ción del Plan como instrumento sistemático de control que, entre otros aspectos, debe recoger indicadores de seguimiento. Esta memoria, como ya exigía la Ley de Ordenación del Territorio de Andalucía, debe ser informada por el órgano administrativo responsable y por la Comisión de Ordenación del Territorio y Urbanismo de Andalucía. Finalmente, las deter- minaciones del POTA también reconocen la participación pública como mecanismo para desarrollar y potenciar en los procesos de planificación y gestión del territorio. Todas estas medidas, entre otras, se encuentran a su vez dentro del Plan de Actuación del POTA, recogidas en el apartado de "Actuaciones de Seguimiento y Gestión” (Tabla 2).

Tabla 2. Actuaciones de seguimiento y gestión previstas por el Plan de Ordenación del Territorio de Andalucía

SG01- Sistema de Información Territoral

SG02- Seguimiento y evaluación: informe de seguimiento continuo de realidad territorial

SG03- Sistema de indicadores para la evaluación de la planificación territorial

SG04- Sistema de información de espacios y bienes catalogados del patrimonio territorial

SG05- Programa de cartografía sobre riesgos

SG06- Cauce de participación municipal

Fuente: elaboración propia a partir del Plan de Ordenación del Territorio de Andalucía de 2006 (2007).

Como valoración final podemos decir que la realidad de los mecanismos establecidos por el POTA es que no aparecen consolidados en su mayoría, muchas de estas actuaciones parecen quedarse en unas buenas intenciones. El proceso de evaluación de los planes no parece ser una de las partes más destacables en la implementación de los planes de ordenación del territorio (Benabent, 2009 y 2012). La falta de consolidación destaca especialmente en el Observatorio Territorial de Andalucía previsto en la Ley, pieza que debería ser clave en el seguimiento y evaluación del desarrollo territorial en la región. Aunque fue creado por Orden de 13 de marzo de 2007 de la Junta de Andalucía, actualmente no ha sido desarrollado ni está en funcionamiento. Sin embargo, se pueden reconocer ciertas licitaciones en relación con el Sistema de Información Territorial y su mejora, así como otros trabajos fuertemente relacionados con este sistema, entre los que destaca y con mayor relevancia los vinculados al Instituto de Estadística y Cartografía de Andalucía, a través de iniciativas como el Sistema de Información Multiterritorial 
de Andalucía. También merece la pena nuevamente mencionar que en relación con la participación pública prevista por el POTA como elemento para desarrollar en la planificación y gestión territorial, se aprecia una intención de información e intercambio de conocimiento, más que una clara apuesta por implicar a la población en la toma de decisiones.

Atendiendo a los planes de ordenación territoriales subregionales andaluces sometidos a las determinaciones del POTA, lo primero que podemos comprobar es que en ellos se establecen las ya citadas comisiones de seguimiento mediante el mismo decreto normativo. Por otra parte, existe una gran diferencia entre los planes de mayor antigüedad y los más recientes a la hora de abordar sus mecanismos previstos de evaluación y seguimiento. Una gran cantidad de los planes aprobados de forma definitiva no presenta indicaciones de seguimiento, solamente muestra algunas referencias en relación con la normativa vinculada a los informes y memorias de seguimiento del plan que, en teoría, deben efectuarse en un número determinado de años pero que raramente son considerados como debieran.

La entrada en vigor de la Ley $7 / 2007$ de 9 de julio, de Gestión Integrada de la Calidad Ambiental (GICA) ha supuesto un antes y un después en los planes de ordenación del territorio aprobados, puesto que es a par- tir de esa fecha cuando se encuentra explícitamente la formulación de indicadores de seguimiento de los planes elaborados a través de sus informes de sostenibilidad ambiental. Lo cierto es que todos los informes de sostenibilidad ambiental plantean prácticamente unos contenidos comunes, incluyendo el sistema de indicadores de seguimiento que solo varía puntualmente en algunos planes. La adaptación de los instrumentos al ámbito de aplicación como normativa del POTA (art. 171) no parece del todo conseguida en este aspecto, pero sí que se tiende a formular sistemas de indicadores con unas características muy deseables como la homogeneidad y la comparabilidad. Además, los indicadores son presentados como propuestas iniciales, agrupados por temáticas, y casi siempre como indicadores abiertos para adaptarse o modificarse en teoría según transcurra el desarrollo del propio plan de ordenación territorial. Por tanto, esto es coherente con las premisas de evaluación y actualización del sistema de indicadores anunciado por el POTA. Merece finalmente la pena destacar que en los contenidos de los planes de ordenación territorial de ámbito subregional tampoco se presentan unos proyectos de participación pública ambiciosos como ya venimos indicando a lo largo de todo el texto. Comúnmente estos se ciñen a la participación a través de los periodos de información pública establecidos legalmente y con una voluntad nor- 
mativa de implicación de la sociedad en el proceso, aunque en la praxis la participación en la toma de decisiones territoriales es complicada.

Por su parte, el nuevo Plan de Protección del Corredor Litoral de Andalucía, en principio, deberá contener los mismos mecanismos de evaluación y seguimiento que las figuras de planificación territorial anteriores, incluyendo el informe de sostenibilidad ambiental con sus medidas e indicadores de seguimiento y evaluación, como establece la legislación sobre evaluación ambiental de planes y programas en Andalucía.

Finalmente, aparte de los elementos encontrados en la propia legislación y planificación territorial, existen otras iniciativas que favorecen la información para la evaluación territorial en Andalucía y que merece la pena al menos mencionar. Ya ha sido comentado el esfuerzo realizado por el Instituto de Estadística y Cartografía de Andalucía con el Sistema de Información Multiterritorial en relación con la apuesta del eje transversal territorial de la estadística, más aún tras la fusión del Instituto de Estadística y el Instituto de Cartografía andaluz. Otro elemento importante que está siendo desarrollado por la administración ambiental andaluza con fondos europeos es el Sistema de seguimiento ambiental de planes y programas, y su aplicación SIGPLAN, establecido por la Memoria Ambiental del Programa Operativo del Fondo Europeo de Desarrollo Regional de Andalucía 2007-2013. En la misma línea otro esfuerzo por destacar es el Sistema de información geográfica-ambiental de Andalucía que cuenta ya con un largo recorrido. Además, otras instituciones se han acercado recientemente a la ordenación del territorio como observamos en el mencionado Informe del Defensor del Pueblo de Andalucía en 2009 o el monográfico dedicado a la ordenación del territorio del Informe Económico y Financiero de Andalucía del año 2010. Por último, la academia también ha realizado esfuerzos alternativos para la evaluación del desarrollo territorial andaluz, entre los que debemos destacar la publicación periódica de los Informes de Desarrollo Territorial de Andalucía, ya por su tercera edición, realizado por los investigadores del proyecto de investigación de excelencia, Observatorio de la Cohesión, la Diversidad y el Desarrollo Territorial de Andalucía (ODTA), financiado por la Consejería de Innovación, Ciencia y Empresa de la Junta de Andalucía.

\section{Conclusiones: monitorización continua y participada como reto pendiente}

Con sus dificultades, todos estos mecanismos de evaluación y seguimien- 
to de los planes de ordenación del territorio en Andalucía están encima de la mesa y se debe destacar que poco a poco se va avanzando en la definición e implementación de los mismos, como apuntan los autores que se han citado a lo largo del texto. El fortalecimiento del sistema de evaluación y seguimiento de los planes de ordenación del territorio andaluces y de la gobernanza territorial, debe ser fundamental para garantizar el cumplimiento de los objetivos propuestos. La apuesta por la coordinación en red de estos mecanismos posiblemente sea la más adecuada. Por otra parte, la falta de experiencia en esta materia también obliga a avanzar con precauciones que eviten una falsa gobernanza territorial. Sin embargo, la rápida incorporación en la planificación territorial de las normativas citadas denota ya una experiencia muy positiva en Andalucía. Es ahora un buen momento, una vez asentada esta materia de ordenación del territorio en Andalucía, para fortalecer la gestión de los planes de ordenación del territorio. Por tanto, los sistemas de indicadores institucionalizados, armonizados y definidos de forma participativa resultan un instrumento de vital importancia. La evaluación ambiental estratégica es un camino ya lanzado normativamente desde la Unión Europea e implementado en los instrumentos de planificación de forma inicial en Andalucía, esfuerzo que merece la pena, y puede ser el comienzo de la gestión y evaluación real de los planes de ordenación del territorio. Esto es fundamental para garantizar la continuidad del ciclo de planificación y no desaprovechar esfuerzos. Como ya hemos podido observar, esta Directiva es un gran avance pero no suficiente en el contexto actual de complejidad e incertidumbre. Además debemos añadir el problema de la incidencia de la crisis económica en la ordenación del territorio y en el desarrollo de este tipo de mecanismos de evaluación y seguimiento complejos. Aunque haya voluntad política, nos encontramos en una situación caracterizada por la falta de medios, pero, por otra parte, se podrían desperdiciar los esfuerzos realizados en planificación si no se prevén unas medidas para evaluar y aprender del plan. La falta de implementación y desarrollo del Observatorio Territorial de Andalucía es un claro ejemplo de esto.

Por otra parte, el nuevo Plan de Protección del Corredor Litoral de Andalucía es una apuesta clara por la importancia de la ordenación del territorio como política ideal para la cooperación y la coordinación interadministrativa. Se trata de un esfuerzo en el sistema de planeamiento territorial de Andalucía, fortaleciendo la red de planes territoriales andaluces. 
En definitiva, en el contexto de la gobernanza territorial y de la nueva planificación territorial estratégica, la monitorización ágil y continua de la información es un elemento clave. Todos los avances que hemos visto en Andalucía apoyan la tesis de que es necesaria una mayor voluntad política y mayores recursos en estos momentos tan complicados para avanzar en esta importante materia de forma inteligente. La sociedad $\mathrm{y}$ todos los agentes, instituciones y organismos, pueden reconocer fácilmente la relevancia de la fase de gestión de la planificación, puesto que la evaluación y seguimiento del plan es fundamental para comprender el desarrollo de los objetivos marcados y mejorarlos adaptándose a los cambios y nuevas necesidades. Esto es todavía más importante cuando lo que está en juego es el propio territorio, su sostenibilidad y la calidad de vida de sus habitantes.

\section{Referencias}

Benabent, M. (2009). Los planes de ordenación del territorio en España. De la instrumentación a la gestión. En L. Sánchez \& M.A. Troitiño (Coords). Agua, territorio y paisaje: de los instrumentos programados a la planificación aplicada: $V$ Congreso Internacional de Ordenación del Territorio (pp. 143-158). Málaga: Asociación Interprofesional de Ordenación del Territorio FUNDICOT.

Benabent, M. (2012). Treinta años de ordenación del territorio en el estado de las autonomías. En M. Castañar (Ed.). El planejament territorial a Catalunya a inici del segle XXI (pp. 140-165). Barcelona: Institut d'Estudis Catalans, Societat Catalana d'Ordenació del Territori.

Comisión Europea (1999). Estrategia Territorial Europea. Hacia un desarrollo equilibrado y sostenible del territorio de la UE. Luxemburgo: Oficina de Publicaciones de la Unión Europea.

Comisión Europea (2011). Eurostat regional yearbook 2011. Luxemburgo: Oficina de Publicaciones de la Unión Europea. doi: 10.2785/1392

Constitución Española (1978, 29 de dic.). Boletín Oficial del Estado (311).

Collado, J. C. (2004). La Estrategia Territorial de Navarra. Un caso pionero de aplicación de la Estrategia Territorial Europea. En J. Farinós \& J. Romero (Coords). Ordenación del territorio y desarrollo territorial: el gobierno del territorio en Europa: tradiciones, contextos, culturas y nuevas visiones (pp. 289-312). Gijón, Asturias, España: Trea.

Decreto-Ley 5 (2012, 27 de nov.). De medidas urgentes en materia urbanística y para la protección del litoral de Andalucía. Boletín Oficial de la Junta de Andalucía (233). 
ESPON 2.3.2 PROJECT (2007). Governance of territorial and urban policies from EU to local level. (2004-2006). Retrieved from http://www.espon.eu/main/Menu_Projects/ Menu_ESPON2006Projects/Menu_PolicyImpactProjects/governance.html

Farinós, J. \& Romero, J. (2008). La gobernanza como método para encarar los nuevos grandes retos territoriales y urbanos. Boletín de la Asociación de Geógrafos Españoles, $46,5-9$.

Feria, J. M., Rubio, M. \& Santiago, J. (2005). Los planes de ordenación del territorio como instrumentos de cooperación. Boletín de la Asociación de Geógrafos Españoles, 39, $87-116$.

Fernández-Tabales, A., Pedregal, B., Rodríguez, J. C., Pita, M. F. \& Zoido, F. (2009). El concepto de cohesión territorial: escalas de aplicación, sistemas de medición y políticas derivadas. Boletín de la Asociación de Geógrafos Españoles, 50, 157-172.

Garnåsjordet, P. A., Aslaksen, I., Giampietro, M., Funtowicz, S. \& Ericson, T. (2012). Sustainable Development Indicators: From Statistics to Policy. Environmental Policy and Governance, 22(5), 322-336. doi:10.1002/eet.1597

Hildenbrand, A. (2006). La política de ordenación del territorio de las comunidades autónomas: balance crítico y propuestas para la mejora de su eficacia. Revista de derecho urbanístico y medio ambiente, 40(230), 79-140.

Hildenbrand, A. (2009). La ordenación del territorio en la Agenda Política Europea. En L. Sánchez \& M.A. Troitiño (Coords). Agua, territorio y paisaje: de los instrumentos programados a la planificación aplicada: V Congreso Internacional de Ordenación del Territorio (pp. 121-141). Málaga: Asociación Interprofesional de Ordenación del Territorio FUNDICOT.

Juaristi, J. (2009). La ordenación del territorio en el umbral del año 2010: promesas, retos y problemas. Lurralde: Investigación y espacio, 32, 361-382.

Ley de Regulación y Uso del suelo y Ordenación Urbana (1956, 14 de mayo). Boletín Oficial del Estado, (1335).

Ley 1 (1994, 11 de enero). De ordenación del territorio de la comunidad autónoma de Andalucía. Boletín Oficial de la Junta de Andalucía, (8), (22 de enero de 1994).

Ley 9 (2006, 28 de abril). Sobre evaluación de los efectos de determinados planes y programas en el medio ambiente (vigente hasta el 12 de diciembre de 2013). Boletín Oficial del Estado, (102), (29 de abril de 2006).

Ley 7 (2007, 9 de julio). De Gestión Integrada de la Calidad Ambiental. Boletín Oficial de la Junta de Andalucía, (143), (20 de julio de 2007).

Massiris, A. (2002). Ordenación del territorio en América Latina. Scripta Nova, Revista electrónica de geografía y ciencias sociales, 6 (125), 105-132. 
Munarriz, D. (2011). El sistema navarro de gobernanza territorial. El papel del Observatorio territorial de Navarra. En J. Farinós, J. Romero \& J. Salom (eds.). Cohesión e inteligencia territorial: dinámicas y procesos para una mejor planificación y toma de decisiones (pp. 215-244). Valencia, España: Publicaciones de la Universitat de València.

Plan de Ordenación del Territorio de Andalucía de 2006. (2007). Sevilla: Consejería de Obras Públicas y Transportes, Junta de Andalucía.

Romero, J. \& Farinós, J. (2011). Redescubriendo la gobernanza más allá del buen gobierno. Democracia como base, desarrollo territorial como resultado. Boletín de la Asociación de Geógrafos Españoles, 56, 295-319.

Segura, S. \& Pedregal, B. (2011). Indicadores de seguimiento y control en planes de ordenación del territorio. En V. Gonzálvez \& J.A. Marco (eds.). Urbanismo expansivo: de la utopía a la realidad. XXII Congreso de Geógrafos Españoles (Asociación de Geógrafos Españoles) (pp. 691-702). Alicante, España: Universidad de Alicante.

Zarraluqui, L. (2003). La Estrategia Territorial de Navarra, primera experiencia en España de aplicación de los principios de planificación y desarrollo espacial europeos a un nivel regional. Urban, 8, 111-122.

Zoido, F. (2010). Ordenación del territorio en Andalucía: reflexión personal. Cuadernos Geográficos de la Universidad de Granada, 47, 189-221. 
Recepción: 18 de octubre de 2013

Evaluación: 9 de marzo de 2014

Aprobación: 10 de octubre de 2014 